KOMPASS

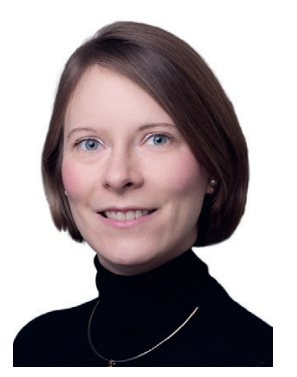

Nina Rosa Neuendorff

Koordination «OnkoCampus»

\title{
Serien abschließen, Studien starten
}

Liebe Leserinnen und Leser,

herzlich willkommen! Wir hoffen, Ihnen auch in der aktuellen Ausgabe wieder interessante Themen präsentieren zu dürfen!

In der nun vorliegenden Ausgabe schließen wir unsere Serie zur Fertilität mit 2 Beiträgen ab. Herr Prof. Nawroth stellt das FertiProtekt-Netzwerk vor, eine Initiative, die insbesondere den zeitnahen Zugang zur Fertilitätsberatung und - Protektion in den letzten Jahren in Deutschland erheblich verbessert hat. Ein weiterer Schwerpunkt der aktuellen CampusAusgabe stellt das Thema «Testosteronsubstitution nach Orchiektomie» dar. Wir danken Frau Professor Kliesch, Münster, und Herrn Maghaireh, Würzburg, für eine Praxisanleitung zu diesem wichtigen Thema, das insbesondere die Lebensqualität, aber auch viele andere präventivmedizinische Aspekte orchiektomierter Patienten doch entscheidend verbessern kann. Frau Dr. Maria Lipp und Frau Prof. Katharina Hancke, die wir auch schon in den vergangenen Ausgaben für unsere Fertilitätsserie als Autorinnen gewinnen konnten, runden das Thema des Fertilitätserhaltes nun mit einer Übersicht zur Kryokonservierung bei Männern ab.

Ein weiterer Themenschwerpunkt stellt die Initiierung klinischer Studien dar. Prof. Richard Schlenk vom Nationalen Tumorzentrum (NCT) in Heidelberg erzählt von seiner Begeisterung für seine Tätigkeit.

Nun wünsche ich Ihnen viel Spaß beim Lesen und scheuen Sie sich nicht, uns bei Anregungen oder Kritik zu kontaktieren, vielleicht gibt es ja auch Themenwünsche?

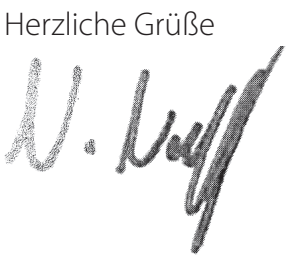

Nina Rosa Neuendorff

\section{KARGER}

Fax +497614520714 information@karger.com www.karger.com
(C) 2019 S. Karger GmbH, Freiburg
Dr. Nina Rosa Neuendorff

Medizinische Klinik V (Hämatologie, Onkologie und Rheumatologie)

Universitätsklinik Heidelberg

Im Neuenheimer Feld 410, 69120 Heidelberg

NinaRosa.Neuendorff@med.uni-heidelberg.d 


\section{Testosteronsubstitution nach Orchiektomie}

Eine Orchiektomie, die Entfernung eines oder beider Hoden, wird in der Mehrzahl der Fälle im Rahmen einer malignen Keimzelltumorerkrankung durchgeführt, um den erkrankten Hoden zu entfernen. Bei bis zu 9\% der Betroffenen kann der Hodentumorbefall beidseitig vorliegen. Im Einzelfall kann eine organerhaltende Tumorenukleation des zweiten kontralateralen Hodens erfolgen. Liegt nur eine Tumorvorstufe (Keimzellneoplasie des Hodens) des kontralateralen Hodens vor oder wurde eine hodenerhaltende Tumorenukleation durchgeführt, muss der Resthoden im Regelfall bestrahlt werden. Selten führen testikuläre Metastasen zum Organverlust. Darüber hinaus können auch die systemische Therapie oder eine Strahlentherapie onkologischer Erkrankungen zum testikulären Funktionsverlust und damit nicht nur zu Zeugungsfähigkeit, sondern auch zum Ausfall der Testosteronproduktion führen.

Auch benigne Erkrankungen können zur Orchiektomie führen, beispielsweise aufgrund von Stromatumoren, entzündlichen Prozessen, Durchblutungsstörungen nach Hodentorsion oder infolge von traumatischen Prozessen. Im Rahmen einer Korrektur eines Maldeszensus kann es ebenfalls zum Organverlust kommen. Extrem selten ist die angeborene Anorchie.

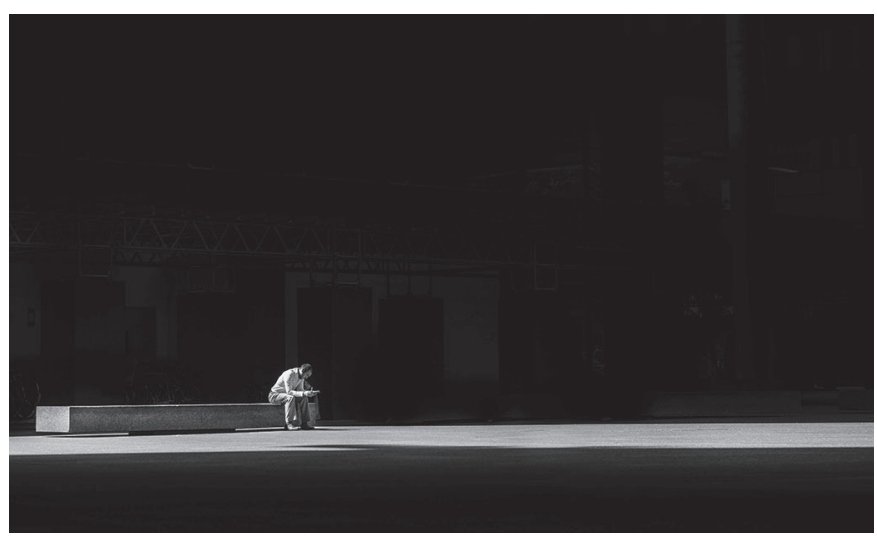

Die Therapie onkologischer Erkrankungen kann neben Zeugungsunfähigkeit auch den Ausfall der Testosteronproduktion zur Folge haben.

Bei allen Zuständen, die mit einem (Funktions-)Verlust beider Hoden einhergehen, muss spätestens ab Beginn des Zeitpunktes des normalen Pubertätseintrittes eine Testosteronsubstitution erfolgen. Die überwiegende Zahl der Betroffenen erlebt einen Hodenverlust im Erwachsenenalter postpubertär. Die Leitlinie der Europäischen Gesellschaft für Urologie zum «Männlichen Hypogonadismus», die zuletzt 2018/2019 aktualisiert wurde, weist den Keimzelltumor des Hodens als eine der häufigsten Ursachen des Hypogonadismus aus. Die nachfolgenden Ausführungen geben im Wesentlichen die in dieser Leitlinie zusammengefasste Evidenz und die entsprechenden Empfehlungen wieder [1].

Eine Testosteronsubstitution ist indiziert bei verminderten Testosteronserumspiegeln, die das Gesamt- und/oder das freie Testosteron betreffen, und mit Symptomen des Testosteronmangels einhergehen. Das Krankheitsbild nach einer Orchietkomie ist der sogenann- te primäre Hypogonadismus, dem eine testikuläre Unterfunktion oder der vollständige Ausfall der Leydig-Zellen zugrunde liegt. Die Symptome sind abhängig vom Alter bei Manifestation des Hypogonadismus und dem Ausmaß des Testosterondefizits. Physiologische Testosteronserumspiegel beim Erwachsenen liegen in den Morgenstunden bei $\geq 12 \mathrm{nmol} / \mathrm{l}$ Gesamttestosteron und $\geq 250 \mathrm{pmol} / \mathrm{I}$ freies Testosteron (fT), wobei das fT durch die Bestimmung des SHBG und eine Kalkulationsformel (nach Vermeulen) ermittelt wird [2]. Die Bestimmung sollte mindestens zweimal im Abstand weniger Wochen morgens vor 11 Uhr und möglichst nüchtern erfolgen. Die häufigsten Symptome eines Hypogonadismus beim postpubertären Mann sind somatischer, sexueller und kognitiv-psychischer Qualität und können unterschiedlich stark variieren.

Die Substitution hat das Ziel, physiologische Testosteronserumspiegel wieder herzustellen und die Symptome zu lindern bzw. die physiologische Situation wieder herzustellen. Für die Therapie stehen transdermale und intramuskulär injizierbare Testosteronpräparate zur Verfügung. Die Auswahl des Präparates hängt wesentlich von den Präferenzen des Patienten ab. Die transdermal anzuwenden Gelpräparate müssen zuverlässig täglich aufgetragen werden, die Resorption kann durch den Hauttyp und die Konzentration des Gels (1\%ig bis 2,5\%ig) beeinflusst werden. Eine Kontamination von Frauen und Kindern ist unbedingt zu vermeiden. Die regelmäßige morgendliche Applikation imitiert die natürliche zirkadiane Rhythmik und eine problemlose Dosisanpassung ermöglicht eine sehr individuelle und rasche Therapiesteuerung zum Erreichen physiologischer Serumspiegel. Die intramuskuläre Anwendung differenziert zwischen einem kürzer und länger wirksamen Testosteronester. Das kurzwirksame Präparat (z.B. Testosteronenantat 250 mg), das alle 2-3 Wochen injiziert werden muss, erreicht aufgrund seiner hohen Fluktuation der Wirkspiegel unmittelbar nach Injektion das Ziel einer modernen und physiologischen Substitution nicht vollständig. Es ist jedoch das einzige Präparat, das auch im Fall der Substitution für unter 18-jährige Patienten zugelassen ist. Für das nach aktuellem Wissensstand geforderte Erreichen von physiologischen Zielwerten zur Vermeidung von unerwünschten Nebenwirkungen, steht für die intramuskuläre Injektion Testosteronundecanoat (1000 mg) zur Verfügung. Dieses Präparat muss 6-8 Wochen nach der Erstinjektion zum zweiten Mal injiziert («aufgesättigt») werden. Danach wird die Injektion alle 12 Wochen (10-14 Wochen) erneuert. Vor Einleitung der Therapie müssen mögliche Kontraindikationen gegen eine Testosterontherapie abgeklärt bzw. ausgeschlossen werden:

- Unbehandeltes, lokal fortgeschrittenes oder metastasiertes Prostatakarzinom

- Viriles Mammakarzinom

- Männer mit aktivem Kinderwunsch

- Hämatokrit > 0,54 (bzw. Erkrankungen, die mit Polyglobulie einhergehen)

- Schwere chronische Herzschwäche (Klasse IV der New York Heart Association) 
Bei Einleitung der Therapie muss der Patient darüber aufgeklärt sein, dass es unterschiedlich lange dauern kann, bis Symptome sich verändern oder verschwinden. Während Wohlbefinden, Libido und Potenz bereits nach 3 Monaten spürbare Verbesserungen erfahren können, zeigen sich metabolische Effekte oder Effekte auf den Knochenstoffwechsel erst nach 6-12 Monaten. Unter der Substitution sollte nach 3 Monaten eine erste Kontrolle erfolgen, um die Höhe des Testosteronspiegels zu kontrollieren und - bei den Gelpräparaten - gegebenenfalls eine Dosisanpassung vorzunehmen. Bei der Injektionstherapie ist eine Spiegelkontrolle im Blut erst am Ende des Injektionsintervalles nach der Aufsättigung sinnvoll (also entweder nach 18 Wochen oder nach der 3. Injektion nach weiteren 12 Wochen). Im ersten Therapiejahr erfolgt die weitere Kontrolle dann nach 6 und 12 Monaten und danach in jährlichen Intervallen. Zusätzlich zur Labordiagnostik erfolgt bei den Kontrollterminen das Abfragen der klinischen Symptome, gegebenenfalls ergänzt durch Fragebögen und Abfrage des Befindens des Patienten. Besonderes Augenmerk bei über 45-jährigen Patienten ist auf die Prostata zu richten, weswegen auch der PSA-Wert und eine digital rektale Prostatapalpation zur jährlichen Untersuchung gehören. Da Testosteron auch die Erythropoese stimuliert, gehört zu den Follow-up Untersuchungen auch die Blutbildbestimmung, um rechtzeitig einen zu hohen Hämatokritwert und damit ein erhöhtes Thromboserisiko zu erkennen. Bei einem Hämatokritwert > 0,54 wird die Dosis (vorübergehend) reduziert oder das Injektionsintervall verlängert, gegebenenfalls muss ein Aderlass erfolgen.

Selten sind Symptome und Testosteronserumwerte nicht in Einklang zu bringen. Dann sollte die Diagnose des Hypogonadismus erneut überprüft und auch an die Möglichkeit einer verminderten Androgenrezeptor-Empfindlichkeit gedacht werden. In diesen Fällen kann die CAG-repeat-Analyse des Androgenrezeptors hilfreich sein, um normal sensible ( $<24$ CAG repeats) oder eher etwas unempfindliche Androgenrezeptortypen ( $\geq 24$ CAG repeats) zu differenzieren und die Therapie anzupassen.

Wird ein hypogonadaler Patient über einen längeren Zeitraum nicht substituiert, werden die erniedrigten Testosteronserumspiegel zu einer Verstärkung der Symptome führen, die individuell unterschiedlich stark vom Patienten wahrgenommen werden. Insbe- sondere Veränderungen der Knochendichte (Osteopenie, Osteoporose) oder eine Verschlechterung der Insulinresistenz können vom Patienten nicht selbst wahrgenommen werden. Eine Anämie kann sich in vermehrter Müdigkeit und Erschöpfung, verminderter Leistungsfähigkeit und -bereitschaft äußern, ein Verlust der körperlichen Kraft ist ein Hinweis auf die Abnahme der Muskelmasse und eine Zunahme des viszeralen Bauchfettes spiegelt die ungünstigen metabolischen Veränderungen wider. Testosteron ist nicht nur das wichtigste Sexualhormon des Mannes, sondern auch ein wichtiger Modulator verschiedener Organfunktionen. Ein ausgeglichener Testosteronhaushalt lindert nicht nur akute Testosteronmangelsymptome, sondern beugt auch Spätschäden vor. Langzeitdaten von unbehandelten und substituierten hypogonadalen Patienten im Vergleich zu eugonadalen Männern zeigen eine höhere Morbidität und auch Mortalität für den unbehandelten hypogonadalen Mann.

Kontakt

Prof. Dr. med. Sabine Kliesch, Chefärztin Abteilung Klinische und Operative Andrologie Centrum für Reproduktionsmedizin und Andrologie WHO Kooperationszentrum, EAA Trainingszentrum, Universitätsklinikum Münster, Domagkstraße 11, 48149 Münster, Deutschland, kliesch@ukmuenster.de, andrologie@ukmuenster.de

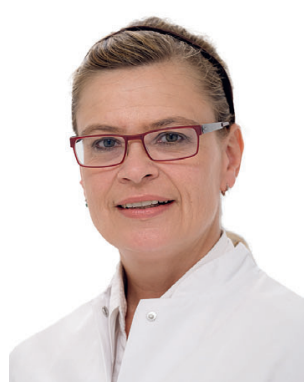

Prof. Sabine Kliesch, Chefärztin der Abteilung Klinische und Operative Andrologie des Universitätsklinikums Münster

\section{Literatur}

1 Dohle, G.R., S. Arver, C. Bettocchi, T.H. Jones, S. Kliesch. EAU-Leitlinie Männlicher Hypogonadismus. J Reproduktionsmed Endokrinol_Online 2018. 15(2):71

2 Bhasin, S., et al. Reference ranges for testosterone in men generated using liquid chromatography tandem mass spectrometry in a community-based sample of healthy nonobese young men in the Framingham Heart Study and applied to three geographically distinct cohorts. J Clin Endocrinol Metab, 2011. 96: 2430. 


\section{Testosteronsubstitution: Eine Übersicht}

\section{Wer muss substituiert werden?}

Grundsätzlich jeder Patient mit symptomatischem Hypogonadismus muss substituiert werden. Einer der häufigen Ursachen für den primären Hypogonadismus ist die uni- oder bilaterale Orchiektomie, meist aufgrund von Hodenkarzinomen, seltener durch Trauma, Infektion oder nach abgelaufener Hodentorsion. Ein gesunder Einzelhoden ist in der Regel in der Lage, einen physiologischen Testosteronspiegel zu produzieren, allerdings ist dabei das Risiko, einen Hypogonadismus zu entwickeln, höher. Beispielsweise haben Patienten mit Einzelhoden nach Orchiektomie wegen eines Hodenkarzinoms ein im Vergleich zu gesunden Männnern vierfaches (altersadaptiertes) Risiko, einen Hypogonadismus zu entwickeln. Das Risiko steigt bei adjuvanter Radio- oder Chemotherapie. [1]

Nach bilateraler Orchiektomie sinkt das Testosteron unter der Kastration innerhalb der ersten 12 Stunden (Median: 8.6 Stunden), sodass die Substitution unmittelbar postoperativ erfolgen sollte. [2] Die ISA, ISSAM, EAU, EAA and ASA haben folgende Empfehlungen bezüglich der Testosteron-Bestimmung und Substitution bei Männern mit Hypogonadismus getroffen:

- Testosteron-Bestimmung sollte nur bei symptomatischen Patienten durchgeführt werden

- Die Blutentnahme sollte aufgrund der zirkadianen Rhythmik des Testosterons zwischen 8 und 11 Uhr morgens erfolgen.

- Bei Gesamttestosteron > $12 \mathrm{nmol} / \mathrm{l}(3,5 \mathrm{ng} / \mathrm{ml})$ sollte nicht substituiert werden und nach anderen Ursachen der Symptome gesucht werden

- Bei Gesamttestosteron < $8 \mathrm{nmol} / \mathrm{l}(2,3 \mathrm{ng} / \mathrm{ml})$ sollte beim symptomatischen Patienten substituiert werden

- Bei Gesamttestosteron zwischen $8 \mathrm{nmol} / \mathrm{l}$ und $12 \mathrm{nmol} / \mathrm{l}$ sollte das freie Testosteron (bioverfügbares Testosteron) bestimmt werden; bei Wert < 200 pmol/I sollte die Substitution erfolgen, andernfalls besteht kein Androgen-Defizit.

\section{Wer sollte nicht substutiert werden?}

\section{Absolute Kontraindikationen:}

- Unbehandeltes oder fortgeschrittenes Prostatakarzinom

- Verdacht auf Mammakarzinom

- Aktueller Kinderwunsch

- Unklare Polyzythämie

Relative Kontraindikationen:

- Geringgradige Polyzythämie

- Wasserretention

- Unklare Gynäkomastie

\section{Wie wird substituiert?}

\begin{tabular}{lll}
\hline Administration & Substanz & Dosis \\
\hline Transdermal & Testosteron-Gel & $50-100 \mathrm{mg} / \mathrm{d}$
\end{tabular}

Vorteile: konstante physiologische Testosteronspiegel, einfache Anpassung der Dosierung oder Absetzung bei Unverträglichkeit; Nachteile: tägliche Anwendung

\begin{tabular}{lll}
\hline Intramuskulär & Testosteronunde- & Zweitinjektion 6-8 Wochen \\
& canoat & nach Erstinjektion («aufsättigen») \\
& Danach Injektion alle \\
& $10-14$ Wochen
\end{tabular}

Vorteile: langes Therapieintervall;

Nachteile: Supraphysiologische Testosteronserumkonzentration unmittelbar nach Injektion und infraphysiologische Konzentration am Ende des Therapieintervalls

\begin{tabular}{|c|c|c|}
\hline $\begin{array}{l}\text { Oral } \\
\text { (Ineffektive Substitu- } \\
\text { tion und deshalb }\end{array}$ & $\begin{array}{l}\text { Testosteron } \\
\text { Undecanoat }\end{array}$ & $\begin{array}{l}2 \text { Kapseln, } 2-3 \text { mal/d mit } \\
\text { einer fettreichen Mahlzeit } \\
(120-200 \mathrm{mg} / \mathrm{d})\end{array}$ \\
\hline
\end{tabular}
heute obsolet)

Nachteile: kurzfristige Schwankungen des Serumtestosterons erfordert eine hohe Patientencompliance

\section{Wie wird die Therapie überwacht?}

- Testosteron \& LH: alle 3 Monate, bei insuffizienter Substitution sollte die Dosierung erhöht bzw. die Therapieintervalle verkürzt werden.

- Blutbild: alle 3 Monate, bei einem Hämatokrit von > 0,54 wird die Dosis (vorübergehend) reduziert oder das Injektionsintervall verlängert.

- Prostata-Vorsorge mit PSA und DRU alle 6 Monate, bei Verdacht auf Prostatakarzinom sollte die Substitution pausiert werden. Beim Nachweis eines Prostatakarzinoms beim hypogonadalen Mann gestatte die Europäische Gesellschaft für Urologie die Substitution nur bei lokal begrenztem, Niedrig-Risiko Prostatakarzinom und frühestens ein Jahr nach der Behandlung des Karzinoms.

- Knochendichtemessung (Densitometrie): alle 2 Jahre, bei Osteoporose sollte die Therapie zusätzlich um Calcium und Vitamin D ergänzt werden.

Kontakt: Omar Maghaireh, Funktionsoberarzt Klinik für Urologie und Kinder Urologie, Universitätsklinikum Würzburg, Würzburg, Deutschland, omarmaghaireh@hotmail.com

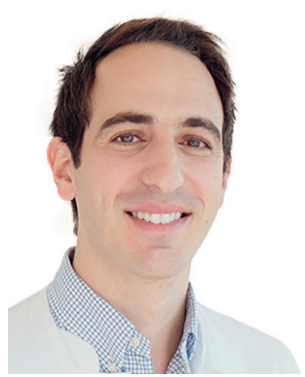

Omar Maghaireh, Funktionsoberarzt der Klinik für Urologie und Kinder Urologie am Universitätsklinikum Würzburg

Die Literaturangaben finden Sie unter www.karger.com/?DOl=502246 
Fertilität und Krebserkrankung

\section{Spermien und Hodengewebe konservieren}

Als fertilitätserhaltende Maßnahme beim Mann steht die Kryokonservierung von Spermien zur Verfügung. Etwa 50\% der eingefrorenen und später aufgetauten Spermien verlieren durch diese Prozesse ihre Vitalität. Die Verwendung der Spermien ist in den meisten Fällen nur durch eine Intrazytoplasmatische Spermieninjektion (ICSI) sinnvoll möglich. Bei Azoospermie oder falls (z.B. posttraumatisch) keine Ejakulation möglich ist, können mittels mikrochirurgischem Eingriff Spermien aus operativ freigelegtem Hodengewebe entnommen und anschließend kryokonserviert werden (sogenannte TESE = Testikuläre Spermienextraktion). Bei prä- oder frühpubertären Jungen, deren Spermatogenese noch nicht vollständig abgeschlossen ist, kann Hodengewebe mit den sich darin befindenden spermatogonialen Stammzellen entnommen und kryokonserviert werden. Dieser Ansatz ist jedoch rein experimentell [1]. Praktisches Vorgehen: Idealerweise sollte der Patient die Spermien vor Ort im Kinderwunschzentrum abgeben, da die Qualität durch den Transport in der Regel leidet. Falls die Abgabe vor Ort in einem Zentrum nicht möglich ist (aus physischen oder psychischen Gründen) wäre ein Transport von 30-45 min möglich, allerdings sollten die Spermien in einem sterilen Becher und im Idealfall körperwarm transportiert werden. Auf keinen Fall sollten die Spermien gekühlt transportiert werden, da dadurch die Qualität abnimmt und die Chancen für eine spätere Schwangerschaft nicht mehr gewährleistet werden können.

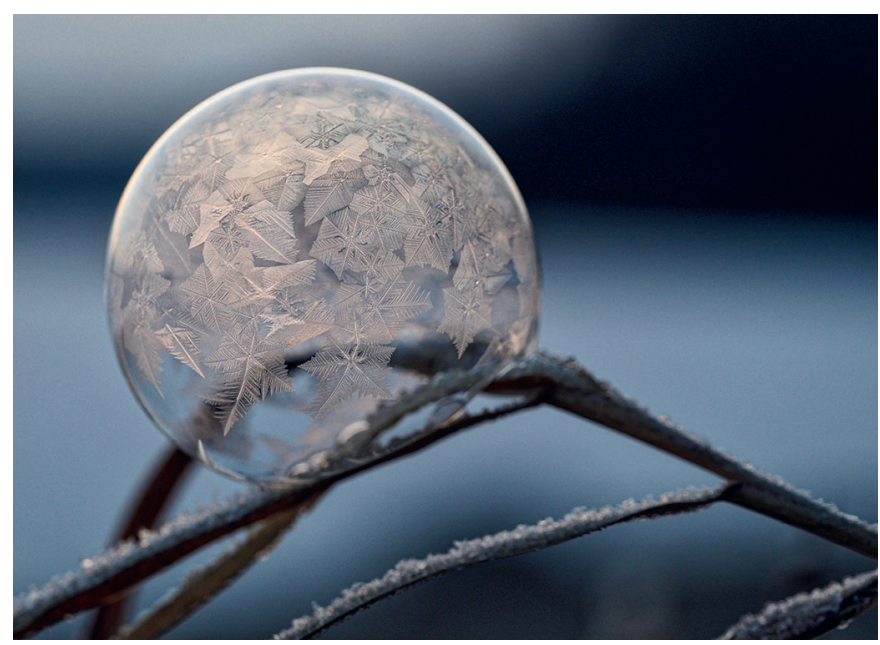

Einfrieren und Auftauen schadet den Spermien. Rund 50\% verlieren die Vitalität.

Kontakt

Dr. Maria Lipp, Universitätsfrauenklinik UIm, Prittwitzstraße 43, 89075 Ulm, Deutschland, maria.lipp@uniklinik-ulm.de

\section{Literatur}

1 M. Balcerek et al., Perspektive Fertilität Indikation und Durchführung fertilitätsprotektiver Massnahmen bei onkologischen und nicht-onkologischen Erkrankungen, 1st ed. Kiel: von Wolff, M., 2016.

\section{Fertilität und Krebserkrankung \\ Das FertiProtekt Netzwerk stellt sich vor}

Der Beratungsbedarf zur Fertilitätsprotektion stieg in den letzten Jahren relevant - eine Folge der immer besseren Überlebensraten bei onkologischen Erkrankungen und der optimierten fertilitätserhaltenden Maßnahmen. In vielen Fällen ist die Zeit, die zur Beratung und gegebenenfalls nachfolgenden protektiven Therapie zur Verfügung steht, ein bedeutender Faktor. Daher stellen logistische Netzwerke wie FertiPROTEKT eine optimale Voraussetzung dar, um die Beratung und fertilitätsprotektiven Konzepte möglichst schnell umzusetzen.

\section{Entwicklung des FertiPROTEKT Netzwerk e.V.}

Bis Anfang des Jahrtausends resultierten aus einzelnen deutschen Arbeitsgruppen bereits wissenschaftliche Untersuchungen und Publikationen zur Fertilitätsprotektion [1, 2], ohne dass eine konzertierte flächendeckende Beratung und Betreuung der Patientinnen etabliert war.

Im Mai 2006 trafen sich auf Initiative und Einladung von Prof. Michael von Wolff (Gynäkologe und Reproduktionsmediziner, damals Abteilung für Gynäkologische Endokrinologie und Fertilitätsstörungen der Universität Heidelberg) sowie Prof. Markus Montag (Biologe, damals Abteilung für Gynäkologische Endokrinologie und Reproduktions- medizin der Universität Bonn) Vertreter/innen aus 30 universitären reproduktionsmedizinischen Zentren zur Gründung des Netzwerkes FertiPROTEKT in Heidelberg. Sie wählten für jeweils 2 Jahre ein Leitungsteam, in dem sowohl Mediziner/innen als auch Biologen/innen vertreten waren. Später erfolgte die Öffnung des Netzwerkes auch für Mitgliedszentren aus Österreich und der Schweiz (www.fertiprotekt. de, www.fertiprotekt.ch, www.fertiprotekt.at, www.fertiprotekt.com) sowie für private reproduktionsmedizinische Zentren.

Das Netzwerk sollte unter anderem den nachfolgenden Zielen dienen:

- Schaffung flächendeckender Strukturen zur Durchführung von Beratungen und Therapien zur Fertilitätsprotektion,

- fachlich-inhaltliche sowie interdisziplinäre Abstimmung der Beratungen und Therapien zur Fertilitätsprotektion,

- Dokumentation der Beratungen und Therapien in einem Register,

- Initiierung, Durchführung und Unterstützung von Studien sowie der Publikation der Ergebnisse,

- Festlegung von Standards und Publikation von Empfehlungen. Das Netzwerk erlangte national und international schnell hohen Bekanntheitsgrad. Ohne eine anerkannten Rechtsform ergaben sich aber in der Zusammenarbeit mit anderen Fachgesellschaften, bei 


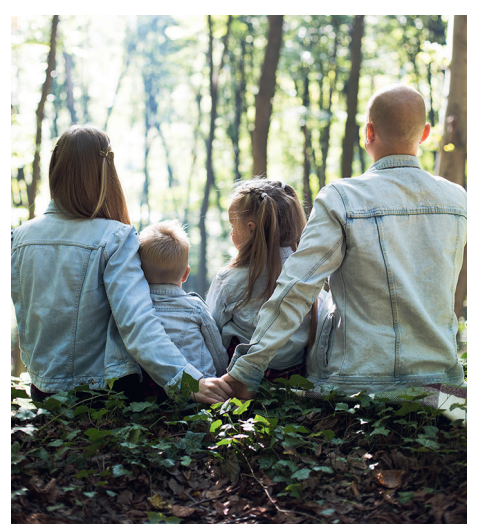

Fertilitätserhalt bei Krebserkrankung: Zeit ist ein wesentlicher Faktor bei der Beratung und nachfolgenden Behandlung.

der Erarbeitung von Leitlinien, oder in der Diskussionen mit Krankenkassen etc. immer zusätzliche Herausforderungen. Daher wurde 2015 beschlossen, FertiPROTEKT in einen eingetragenen Verein zu überführen. Am 10. November 2015 gründete das damalige Leitungsteam in Hamburg den eingetragenen Verein FertiPROTEKT Netzwerk mit Geschäftsstelle in Marburg [3].

Dem Verein gehören zum 24. Juli 2019129 Mitgliedszentren an (Deutschland: 117, Österreich: 8, Schweiz: 4). Mit dem Aufnahmeantrag muss jedes Zentrum nachweisen, dass es alle fertilitätsprotektiven Maßnahmen allein oder ggf. in einer konkret zu benennenden Kooperation vorhält.

Über eine Suchfunktion auf der Homepage lassen sich die aktuellen Mitgliedszentren im Umkreis der/des Suchenden finden, so dass zeitnah eine Betreuung möglich ist.

Der Verein ist Mitglied im Dachverband Reproduktionsmedizin (DVR e.V.), so dass auch auf DVR-Tagungen ab 2019 eine Sitzung des FertiPROTEKT Netzwerk e.V. stattfindet. Daher wurde 2018 beschlossen, die bis zuletzt jährlichen Arbeitstreffen des Netzwerkes nur noch alle 2 Jahre, alternierend mit dem DVR-Kongress, durchzuführen. Die Gestaltung des wissenschaftlichen Programms dieser Arbeitstreffen obliegt dem Vorstand zusammen mit dem die Tagung ausrichtenden Mitgliedszentrum.

Außerdem ist der FertiPROTEKT Netzwerk e.V. mittlerweile kooperierende Gesellschaft der Deutschen Gesellschaft für Gynäkologie und Geburtshilfe e. V.

Alle Beratungen und Therapien wurden von Begin an dokumentiert. Seit einiger Zeit erfolgt die Dokumentation in Kooperation mit dem Deutschen IVF-Register (D.I.R. e.V.), so dass die resultierende statistische Auswertung Ende 2019 erstmals in das D.I.R.- Jahrbuch inkludiert wird.

Die international einmalige Datenerfassung der Zentren ist eine der Ursachen für die gewachsene internationale Reputation von FertiPROTEKT [4]. Diese Datenmenge inklusive der Informationen zu den Therapieergebnissen ermöglicht das Verfassen von Handlungsempfehlungen, ist die Basis für Studien und erlaubt eine nachhaltige Argumentation gegenüber anderen Fachgesellschaften, Kostenträgern, Patientenorganisationen, der Presse, der Politik etc. An der S2kLeitlinie der AWMF «Fertilitätserhalt bei onkologischen Erkrankungen» haben FertiPROTEKT-Mitglieder intensiv mitgearbeitet [5]. Aus der mittlerweile mehr als 13 Jahre andauernden intensiven Arbeit der jeweiligen Leitungsteams/Vorstände zusammen mit den vorbildlichen gemeinsamen Anstrengungen aller Mitgliedszentren resultieren zunehmend flächendeckende Beratungs- und Therapieoptionen, lokale logistische Netzwerke zur optimalen Patientenbetreuung, ein intensiver Austausch zwischen den Zentren sowie zahlreiche Studienaktivitäten und Publikationen [3].

\section{Beispiele für Aktivitäten des Netzwerkes}

Ein wichtiges Ziel des Vereins war und ist die inhaltliche Abstimmung der Beratungs- und Therapieempfehlungen. Als Basis dienen das aktuelle Wissen sowie regelmäßigen Diskussionen unterschiedlicher Themenbereiche im Rahmen z.B. der Arbeitstreffen.

Die ersten Empfehlungen des Netzwerkes zu den Maßnahmen der Fertilitätsprotektion wurden 2011 englischsprachig mit einem «open access» publiziert, um sie einer breiten Leserschaft zugänglich zu machen [6]. Eine umfangreiche Aktualisierung dieser Empfehlungen erfolgte 2018. Um der gewachsenen Komplexität der Thematik gerecht zu werden, stellten die Autoren in einer Arbeit die Indikationen [7] sowie in einer weiteren die Techniken [8] dar. Auch für diese beiden Publikationen finanzierte der Verein den open access.

Auf der Homepage des Vereins findet sich die Anleitung zum kostenfreien Download eines 2016 publizierten Buches [9], dessen überarbeitete Neuauflage 2020 erscheinen wird. Es stellt komprimiert und übersichtlich praxisrelevante Aspekte sowohl der weiblichen als auch männlichen Fertilitätsprotektion bei verschiedenen Erkrankungen dar.

Obwohl das Hauptaugenmerk des FertiPROTEKT Netzwerk e.V. auf der Fertilitätsprotektion bei medizinischen Indikationen liegt, hat sich das Netzwerk darüber hinaus auch anderen Themen gestellt, wenn die Notwendigkeit dazu bestand. Ein Beispiel ist die Positionierung zur - vor allem in der Laienpresse - kontrovers diskutierten Kryokonservierung von unfertilisierten Eizellen ohne medizinischen Grund, dem sogenannten «Social freezing». Das Ergebnis intensiver Diskussionen auf dem Arbeitstreffen 2012 in Hamburg war eine gemeinsame Stellungnahme [10]. Außerdem führte die Darstellung der im Netzwerk zum «Social freezing» erfolgten Beratungen und Therapiezyklen zu einer Veröffentlichung im «Deutschen Ärzteblatt» inklusive Bildgebung für die Titelseite [11].

\section{Kontakt}

Prof. Dr. med. Frank Nawroth, Facharzt-Zentrum für Kinderwunsch, Pränatale Medizin, Endokrinologie und Osteologie, amedes MVZ Hamburg, Hamburg, Deutschland, Frank.Nawroth@amedes-group.com.

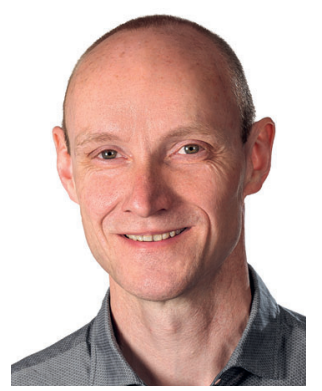

Prof. Dr. Frank Nawroth, Schriftführer des FertiPROTEKT e.V

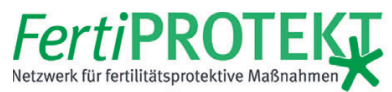

Die Literaturangaben finden Sie unter www.karger.com/?DO/=502246 


\section{Experte im Gespräch}

\section{Medizinische Fragen in Statistik übersetzen}

Aktuelle Studienergebnisse sind wesentlicher Teil der modernen evidenzbasierten Medizin. Aber jeder, der selbst schon einmal an einer Studie beteiligt war, weiß, was für ein Aufwand hinter einer Studie steckt. Wir haben darüber mit Prof. Dr. med. Richard Schlenk gesprochen - er ist oberärztlich tätig in der Klinik für Hämatologie, Onkologie und Rheumatologie sowie der Klinik für Medizinische Onkologie am Universitätsklinikum Heidelberg. Focus seiner Arbeit sind klinische Studien in der Onkologie am Universitätsklinikum Heidelberg sowie am Deutschen Krebsforschungszentrum. Er leitet die Studienzentrale am Nationalen Centrum für Tumorerkrankungen (NCT) in Heidelberg und die Studienzentrale der Klinik für Hämatologie, Onkologie und Rheumatologie und weiß daher genau, wo schon in der Studienvorbereitung die ersten Hürden zu meistern sind.

Karger Kompass Onkologie: Herr Professor Schlenk, beschreiben Sie uns grob, wie so eine Studienplanung abläuft und wie entsprechend Ihr Alltag als Leiter der Studienzentrale am NCT aussieht.

Prof. Richard Schlenk: Mein Arbeitsalltag ist gekennzeichnet durch ein breites Aufgabenspektrum. In Bezug auf die Konzeption klinischer Studien verschaffe ich mir zu Beginn immer einen Überblick über die aktuelle klinische Forschung und molekularbiologischen Erkenntnisse in den Zielpopulationen. Anschließend geht es darum, sich zu überlegen, wie einer Frage, die meine Kolleginnen und Kollegen aufgeworfen haben, in einer klinischen Studie nachgegangen werden kann. Danach geht es weiter mit der Erstellung eines Proposals. Dies umfasst in der Regel 2 bis 3 Seiten und wird verwendet, um potentielle Kooperationspartner, zum Beispiel aus der pharmazeutischen Industrie, zu überzeugen, in die Studie zu investieren, etwa durch die Bereitstellung von Medikamenten, und evtl.. darüber hinaus auch finanzielle Unterstützung für die Durchführung bereitzustellen.

Ist Unterstützung gefunden, wie geht es dann weiter?

Prof. Schlenk: Dann geht es natürlich los: Wir müssen uns überlegen, wie wir die klinische Prüfung umsetzen. Extrem wichtig ist in diesem Zusammenhang das Studienprotokoll. Das stellt den Ablauf der Patientenbehandlung im Rahmen der klinischen Studie dar. Darüberhinaus gibt eine Vielzahl an weiteren Dokumenten, die auf das Studienprotokoll referenzieren und wesentliche Aspekte im Detail beschreiben, zum Beispiel die Kommunikations- und DatenManagement Pläne, der Daten Validierungsplan, der statistsche Analyseplan u.s.w.

Neben den Partnern aus der pharmazeutischen Industrie arbeite ich natürlich auch sehr eng mit Kollegen und Kolleginnen zusammen, die selbst neue Therapeutika entwickeln, z.B bispezifische Antikörper und CAR-T-Zellen im Rahmen des Deutschen Konsortiums für Translationale Krebsforschung. Gemeinsam erarbeiten wir, wie diese neuen Therapieformen bei Patienten umgesetzt werden können und welche klinischen Studien auf dem Weg dahin notwendig sind.
Das klingt tatsächlich nach einem riesigen Planungs- und Koordinationsaufwand...

Prof. Schlenk: Sicher - aber hier hilft mir dann auch die praktische Erfahrung in der Durchführung klinischer Studien am Patienten weiter. Denn erst wer selbst klinische Studien durchgeführt hat, kann sich schon bei der Planung vorstellen, ob etwas im klinischen Alltag umsetzbar ist oder nicht. Und dann ist es auch von Vorteil für mich, dass ich eben nicht nur Mediziner bin, sondern auch Biometriker. Meine zweite Ausbildung habe ich am Institut für Medizinische Biometrie und Informatik in Heidelberg berufsbegleitend gemacht. Das hilft mir sehr, die medizinischen Fragen auch in Statistik umzusetzen.

\section{Welche Fragen sind das dann zum Beispiel?}

Prof. Schlenk: Wir gehen dann zum Beispiel der Frage nach, wie groß der Vorteil einer neuen Therapie gegenüber einer etablierten Therapie sein muss, um diese in einer klinischen Studien zu prüfen und wie viele Patienten wir in einer solchen klinischen Studien behandeln müssen um den Vorteil klar herauszuarbeiten. Oder wie man zeigen kann, dass weniger Nebenwirkungen einen Vorteil für den Patienten bringen bei gleicher Wirksamkeit.

Hat das akademische Umfeld Vorteile für Studien im Vergleich zu Studien in der Industrie?

Prof. Schlenk: Ich weiß nicht, ob man die Frage so einfach stellen kann. Die Studien, die auf der akademischen Seite durchgeführt werden, unterscheiden sich stark von denen in der Industrie. Große Zulassungsstudien etwa werden meist nicht von akademischen Instituten initiiert, sonder von der Pharmazeutischen Industrie durchgeführt. Für diese Studien sind neben dem Prüfplan und der Patienteninformationen sowie -Einverständiserklärung bestimmt 30, 40 oder gar 50 weitere Dokumente notwendig, die jeden auch noch so kleinsten Aspekt einer Studie beschreiben. Das fängt an mit dem Risikomanagementplan, dem Kommunikationsplan, dann geht es weiter mit dem Datenmanagementplan, einem Datenvalidierungsplan. Es gibt für alle Aspekte der Studie Pläne.

In der Industrie werden die Studien also stärker reguliert als im akademischen Umfeld?

Prof. Schlenk: In der Industrie ist jeder Aspekt einer Studie meist vor Start der Studie bereits festgelegt. Langsam kommen wir bei akademischen Studien auch dazu, weil das von den akademischen Sponsoren ebenfalls gefordert wird. Aber man hat bei akademischen Studien immer noch etwas mehr Freiheit. Für mich bietet das noch die Möglichkeit, die Abläufe stärker selbst zu gestalten und das gefällt mir deutlich besser als nach immer dem exakt gleichen vorgegebenen Schema vorgehen zu müssen.

Schränken strikte Regularien die Studien ein?

Prof. Schlenk: Vor kurzem habe ich bei einer Vorlesung in Heidelberg von einer Studie berichtet, die ich 1993 entworfen und durch- 
geführt habe. Damals lagen zwischen Planung und Start der Studie 6 Monate. Das ist eine sehr überschaubare Zeit. Heute dauert das schon eher 2 bis 3 Jahre, wer schneller sein will, braucht einen entsprechenden Apparat und ein entsprechendes Finanzpolster Denn dann müssen viele Leistungen eingekauft werden. Die Regularien schränken uns schon ein und ich denke auch, dass die Anzahl der akademischen Studien durchaus reduziert wird.

Und es gibt da keinen Unterschied in der Kontrolle durch die Behörden, ob akademischer Hintergrund oder Industrie?

Prof. Schlenk: Nein, die Behörden machen keinen Unterschied zwischen der Pharmazeutischen Industrie und einem Universitätsklinikum als Sponsor. Wir hatten vor kurzem eine Inspektion, bei der all diese Pläne, die ich zuvor erwähnt hab, tatsächlich schon bei der Einreichung der Studie erwartet wurden. Und das obwohl man nicht weiß, ob die Studie überhaupt genehmigt wird. Bisher war unsere Herangehensweise meist: Man reicht eine Studie ein und wenn sie genehmigt wird, dann arbeitet man den Rest nach, aber das ist heutzutage ein Auslaufmodell.

Aber nicht nur die Regulierung beeinflusst die Studienlandschaft auch die Schwerpunkte der Forschung ändern sich. Hat das auch Einfluss auf die Studienplanung?

Prof. Schlenk: Ja komplett - in der Onkologie kommen wir immer mehr weg von den einzelnen Krebsentitäten hin zu der Frage: Was steckt denn molekular hinter der Tumorerkrankung? Das führt natürlich dann zu neuen Therapieansätzen und neuen Studiendesignansätzen, wie z.B. Basket-Studien. Die BRAF-Mutation ist bei Melanom häufig aber sie findet sich auch bei der Haarzell-Leukämie. Es macht durchaus Sinn über neue Studiendesigns nachzudenken, wo eben nicht das maligne Melanom Einschlusskriterium ist, sondern BRAF-Mutation. Auf der anderen Seite ist es natürlich so, dass das molekulare Profil der einzelnen Patienten auch relativ einzigar- tig ist. Für die Studienplanung bedeutet das, dass man sich gut überlegen muss, wie man passende Kontrollgruppen schaffen kann. Das ist dann auf der einen Seite sehr spannend und intellektuell sehr herausfordernd. Auf der anderen Seite ist das auch ein Hemmnis, um Studien schnell voran zu bringen.

Was würden Sie jungen Onkologinnen und Onkologen raten, die sich für die klinische Forschung interessieren?

Prof. Schlenk: Da gibt es unterschiedliche Aspekte. Für alle mit einem Faible für Mathematik oder Biologie kann ich durchaus empfehlen, sich über eine biomtrische/statistische Weiterbildung Gedanken zu machen. Das habe ich selbst als intellektuell sehr anspornendempfunden.AufderanderenSeite,wersichfürLaborforschung interessiert und das verknüpfen möchte mit klinischer Forschung, für den ist es wichtig, sich Partner zu suchen, die den anderen Part abbilden können. Insofern sollten sich die jungen Kolleginnen und Kollegen überlegen, was Ihnen am meisten Spaß macht und dann welche komplentären Partner zu Ihnen passen.

Herr Professor Schlenk, viele Dank für das Gespräch und dass Sie sich Zeit für uns genommen haben.

Das Interview führte unser Redakteur Christoph Habel

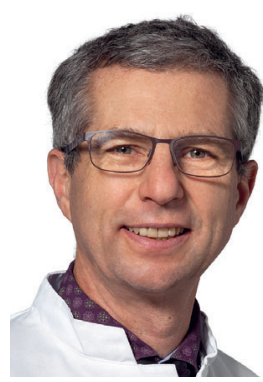

Prof. Richard Schlenk: «Erst wer selbst klinische Studien durchgeführt hat, kann sich schon bei der Planung vorstellen, ob etwas im klinischen Alltag umsetzbar ist oder nicht».

Foto: Universitätsklinikum Heidelberg 